\title{
Electric fields produced by electron irradiation of insulators in a low vacuum environment
}

\author{
M. Toth ${ }^{\mathrm{a})}$ \\ Polymers and Colloids Group, Cavendish Laboratory, University of Cambridge, Madingley Road, \\ Cambridge CB3 OHE, United Kingdom \\ M. R. Phillips \\ Microstructural Analysis Unit, University of Technology, Sydney, Broadway, NSW 2007, Australia \\ J. P. Craven, B. L. Thiel, and A. M. Donald \\ Polymers and Colloids Group, Cavendish Laboratory, University of Cambridge, Madingley Road, \\ Cambridge CB3 OHE, United Kingdom
}

(Received 30 October 2001; accepted for publication 4 December 2001)

\begin{abstract}
We report on the properties of electric fields generated as a result of electron irradiation of dielectrics in a low vacuum scanning electron microscope. Individual field components produced by (i) ionized gas molecules located outside the sample surface and (ii) subsurface trapped charge were detected by measurements of changes in (i) primary electron landing energy and (ii) secondary electron (SE) emission current, respectively. The results provide experimental evidence for a recently proposed model of field-enhanced SE emission from electron irradiated insulators in a low vacuum environment [Toth et al., J. Appl. Phys. 91, 4479 (2002)]. Errors introduced into x-ray microanalysis by the electric fields generated by ionized gas molecules can be alleviated by minimizing the steady state ion concentration by the provision of efficient ion neutralization routes. It is demonstrated how this can be achieved using simple sample-electrode geometries. (C) 2002 American Institute of Physics. [DOI: 10.1063/1.1448876]
\end{abstract}

\section{INTRODUCTION}

Low vacuum scanning electron microscopes (SEMs) are convenient tools for electron imaging and x-ray microanalysis of uncoated insulators. ${ }^{1,2}$ It has long been known that the presence of a partially ionized gas in the specimen chamber alleviates charging artifacts. ${ }^{3}$ SEMs which can tolerate specimen chamber pressures in excess of approximately 4 Torr $(0.5 \mathrm{kPa})$ can sustain liquid $\mathrm{H}_{2} \mathrm{O}$, thus allowing for investigation of unmodified insulating, wet, and entirely liquid specimens. ${ }^{4-6}$ Investigations of such materials have led to reports of contrast in secondary electron (SE) images ascribed to, for example, lateral variations in the dielectric properties of GaN and of entirely liquid emulsion systems, ${ }^{7,8}$ and crystal growth histories in minerals. ${ }^{9}$ Some of the contrast observed in SE images of samples in a low vacuum environment has been ascribed to the electric fields generated by ionized gas molecules (located outside the sample) and by excess charge trapped within insulating specimens. ${ }^{7-11}$ It has been suggested that the electric field generated by gaseous ions can modify the efficiency of gas cascade amplification ${ }^{12}$ and create an SE extraction potential at the sample surface that enhances the emission probability of low energy SEs. ${ }^{11}$ In this article we provide experimental evidence for the existence of such an electric field. It was detected by measuring an increase in the energy of electrons in the electron beam (primary electrons, PEs), at the point of impact on the surface of a dielectric, caused by the transit of PEs through the ion "cloud" in a low vacuum SEM chamber. The PE landing

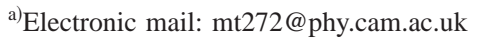

energy was estimated from the maximum bremsstrahlung x-ray energy (the Duane-Hunt limit, DHL) in spectra obtained from dielectrics. ${ }^{13}$ In the case of insulators that contain an excess concentration of negative charge, the presence of two distinct field components generated by (i) positive ions located above the sample surface and (ii) subsurface trapped electrons is demonstrated by the acquisition of (i) $\mathrm{x}$-ray spectra and (ii) SE images, respectively. We discuss the effects of the field produced by ions on SE emission and errors introduced into $\mathrm{X}$-ray microanalysis by the observed changes in PE landing energy. It is demonstrated how the latter can be minimized using sample-electrode geometries under which the steady state ion concentration is limited by provision of efficient ion neutralization paths.

\section{BACKGROUND THEORY}

\section{A. Low vacuum SEM}

A schematic illustration of a low vacuum SEM chamber is shown in Fig. 1. Scattering of electrons in the primary beam by gas molecules leads to the formation of the socalled electron "skirt" around the focused, unscattered component of the electron beam. ${ }^{14,1}$ The skirt can compromise $\mathrm{x}$-ray microanalysis by excitation of regions beyond the nominal beam impact point. ${ }^{1,2,15}$ Electron images can be obtained using the signal induced in a positively biased electrode placed in the specimen chamber. ${ }^{16}$ Common electrode geometries are shown in Fig. 1. The gaseous secondary electron detector (GSED) ${ }^{16,17}$ uses a ring electrode located above the sample surface, centered on the optic axis of the microscope. An array of electrically grounded metallic wires can 


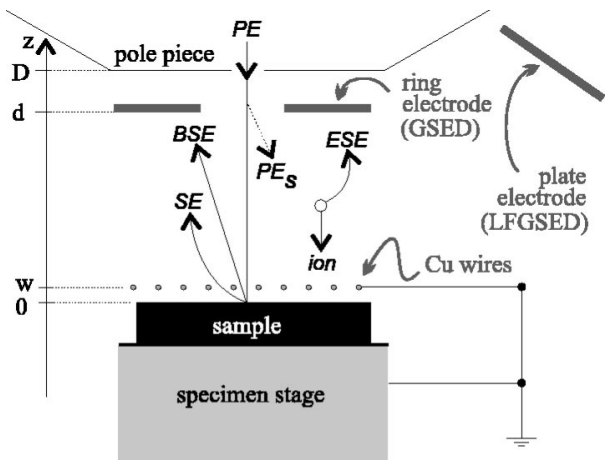

FIG. 1. Schematic illustration of a variable pressure SEM specimen chamber showing the axisymmetric ring electrode [the electron collector of the gaseous secondary electron detector (GSED)], the off-axis plate electrode [the electron collector of the large field GSED (LFGSED)], and an array of electrically grounded $\mathrm{Cu}$ wires located above the sample surface [the wires were only used during the acquisition of data shown in Fig. 10(a)]. The ring or plate electrode was positively biased with respect to the specimen stage (only one gaseous SE detector was installed in the chamber at any one time). Also shown are the directions of motion of charge carriers in the imaging gas: PE:primary electron, $\mathrm{PE}_{s}$ : skirt electron, $\mathrm{BSE}$ : backscattered electron, SE: secondary electron, and ESE: "environmental" SE generated in a gas molecule ionization event $(z=0$ at the sample surface, $D$ : sample-pole piece separation, $d$ : sample-ring electrode separation, and $w$ : sample-Cu wire separation)

be placed above the sample surface in order to provide termination points for some fraction of the electric field originating at the positively biased electrode (this is particularly useful for investigation of bulk dielectric specimens). ${ }^{18}$ The so-called "large field GSED" (LFGSED) uses a rectangular electrode located off-axis, beside the pole piece. ${ }^{17}$

The electric field generated by the bias applied to an electrode (the "detector field") gives rise to a gas ionization cascade that acts as a high gain electron signal amplifier. ${ }^{16,19}$ The gas gain, that is, the number of ion-electron pairs produced by each electron (primary, backscattered, and secondary) injected into the gas can be calculated by assuming a constant electric field between the sample and the electrode. $^{20}$

\section{B. The Duane-Hunt limit (DHL)}

When a sample is irradiated by an electron beam in a high vacuum SEM, the maximum energy of bremsstrahlung $\mathrm{x}$ rays [the so-called "Duane-Hunt limit." (DHL)] excited in the solid is equal to the kinetic energy possessed by PEs when they impact the sample surface (the PE "landing energy" =DHL). ${ }^{13}$ In the case of an electrically grounded conductor, DHL is equal to the energy $\left(\epsilon_{\mathrm{PE}}\right) \mathrm{im}-$ parted to PEs by the SEM accelerating voltage. ${ }^{13}$ If an (uncoated) insulating sample is negatively charged, the electric field generated by excess electrons in the sample extends into the vacuum chamber (and terminates on the pole piece and other conductors in the chamber), decelerates the incoming electron beam, and reduces the PE landing energy. Such negative DHL shifts can be of the order of, but not greater than, $\epsilon_{\mathrm{PE}}$ (i.e., thousands of electron volts). ${ }^{13}$ Conversely, if a sample exhibits positive charging (caused by irradiation by a low energy electron beam, $\epsilon_{\mathrm{PE}} \leqslant \sim 3 \mathrm{keV}$, whereby the emissive current is greater than the injected current) the magni-
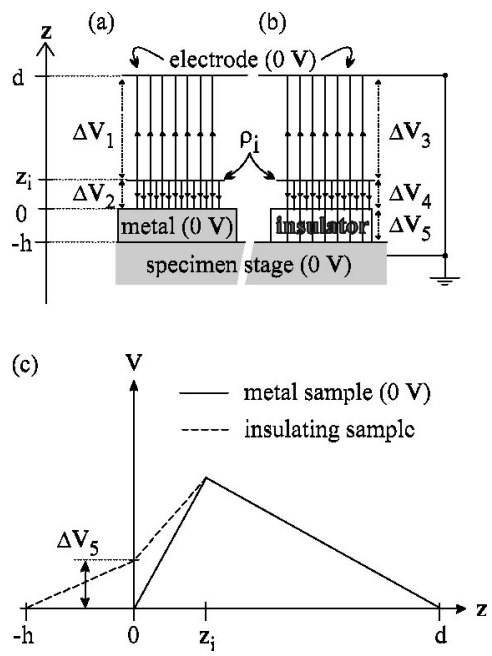

FIG. 2. A simplified illustration of the electric field generated by a plane of positive charge density, $\rho_{i}$, located between two electrically grounded electrodes with (a) a metal and (b) a dielectric specimen placed on the lower electrode. The field generated by $\rho_{i}$ terminates on the upper electrode and on (a) the metal sample and (b) the lower (stage) electrode, respectively. The corresponding potential functions, $V(z)$, are shown in (c).

tude of the field generated by excess holes is self-limited to a few electron volts, due to SE pinning at the sample surface. ${ }^{13}$ Consequently, positive DHL shifts are generally not observed in X-ray spectra obtained in high vacuum SEMs (since $\mathrm{x}$-ray spectrometers employed in SEMs do not have a high enough resolution to detect such small DHL shifts). However, as is discussed below, positive DHL shifts can occur in spectra obtained in a low vacuum environment, ${ }^{21}$ due to the effects of the electric field generated by gaseous ions on the PE landing energy.

It has been suggested that, in a low vacuum SEM chamber, the steady state concentration of ionized gas molecules produced in the gas cascade between the ring electrode and the sample (see Fig. 1) generates an electric field of magnitude that can be a significant fraction of the electrode bias, $V_{e} \cdot{ }^{12,22}$ The distribution of ions within the chamber is determined by the spatial distribution of the ion generation rate and the ion neutralization rate. The latter is a function of the time it takes ions to drift to the sample surface (and chamber walls) and by the electron-ion recombination rate (i.e., the efficiency with which ions capture emitted SEs and electrons in the sample). ${ }^{23}$ The steady state distributions of ions and of the corresponding electric field are presently not known. However, the effects of the field on PE landing energy are governed by the vertical distribution of charge within the sample-electrode gap. This distribution can be represented by a plane of charge density, $\rho_{i}$, located a distance $z_{i}$ above the specimen, as shown in Fig. 2. The plane corresponds to the (vertical) center of gravity of charge within the gas. That is, the height above the sample surface at which the vertical component of the field generated by ions is equal to zero. In the case of an electrically grounded metal specimen, Fig. 2(a), the field generated by $\rho_{i}$ terminates on the upper electrode and on the top surface of the metal sample. Hence the corresponding potential differences between the upper electrode and $z_{i}\left(\Delta V_{1}\right)$, and $z_{i}$ and the specimen surface $\left(\Delta V_{2}\right)$ 


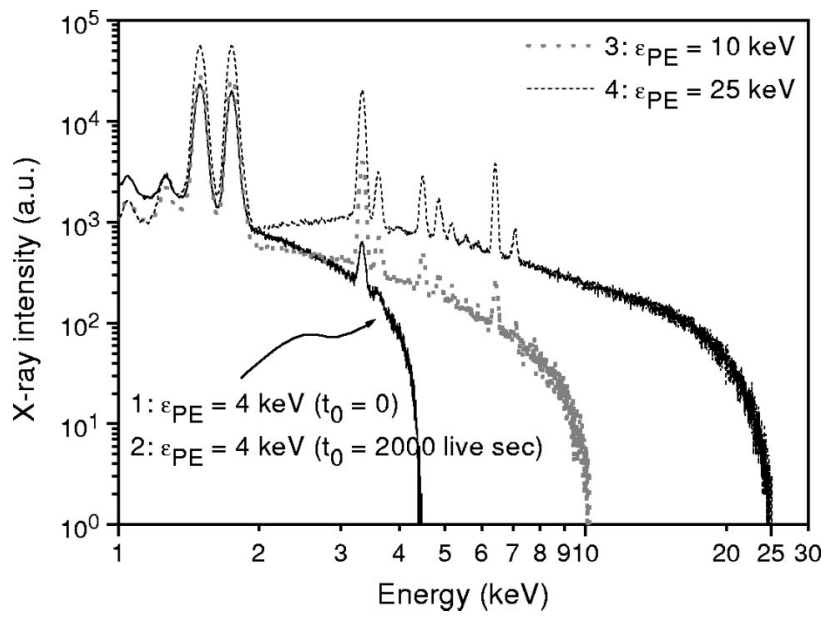

FIG. 3. X-ray spectra acquired consecutively from the same region of mica in the order shown in the figure. The spectra show the dependence of the PE landing energy, DHL, on accelerating voltage. Data obtained under identical conditions (spectra 1 and 2) show the absence of changes in the DHL caused by irradiation-induced sample modification (electron detector $=$ GSED, $P=0.5$ Torr, $V_{e}=550 \mathrm{~V}, D=10 \mathrm{~mm}, d=4.5 \mathrm{~mm}$, horizontal field width $=130 \mu \mathrm{m}$, and $t_{\text {acq }}$ (acquisition time) $=2000$ live s per spectrum).

are equal and opposite: $\Delta V_{1}+\Delta V_{2}=0$. A PE will gain $e \Delta V_{1} \mathrm{~J}$ (where $e$ is the charge of an electron) as it traverses the distance between the top electrode (GSED ring) and $z_{i}$, and will lose the same amount of energy as it travels from $z_{i}$ to the sample surface [see Fig. 2(c)]. The DHL in x-ray spectra obtained from a grounded metal in a low vacuum SEM will therefore be equal to $\epsilon_{\mathrm{PE}}$, as in the case of a high vacuum SEM. Conversely, if the sample is a dielectric, Fig. 2(b), the field generated by $\rho_{i}$ terminates on the specimen stage, not on the sample surface. Consequently, the amount of energy gained by a PE as it travels from the top electrode to $z_{i}\left(e \Delta V_{3}\right)$ is greater than the amount of energy lost as it traverses the distance between $z_{i}$ and the sample surface $\left(e \Delta V_{4}\right)$ since $\Delta V_{3}=\Delta V_{4}+\Delta V_{5}$. Hence PEs will impact the specimen surface with kinetic energy, DHL, given by

$$
\mathrm{DHL}=\epsilon_{\mathrm{PE}}+e\left(\Delta V_{3}-\Delta V_{4}\right)=\epsilon_{\mathrm{PE}}+e \Delta V_{5} .
$$

Positive DHL shifts, $e \Delta V_{5}$, observed in x-ray spectra of insulators are reported in this article.

\section{EXPERIMENT}

All measurements were performed using an FEI Philips XL 30 FEG ESEM equipped with a GSED, LFGSED (see Fig. 1), and an energy dispersive $\mathrm{x}$-ray spectrometer. Water vapor was used as the gas in the specimen chamber. Calibration of the x-ray spectrometer energy scale was verified by acquisition of spectra from grounded Al, in high vacuum (pressure, $P<10^{-6}$ torr), as a function of electron beam accelerating voltage. All $\mathrm{x}$-ray spectra were acquired using an electron beam scan rate of 10 frames/s (TV rate), and using magnifications specified in figure captions by the corresponding horizontal field widths. Each set of spectra acquired as a function of operating parameters (such as $\epsilon_{\mathrm{PE}}$, $V_{e}$, and $P$ ) was obtained more than once to ensure that trends in changes between consecutive spectra did not result from sample modification caused by electron irradiation (ex-
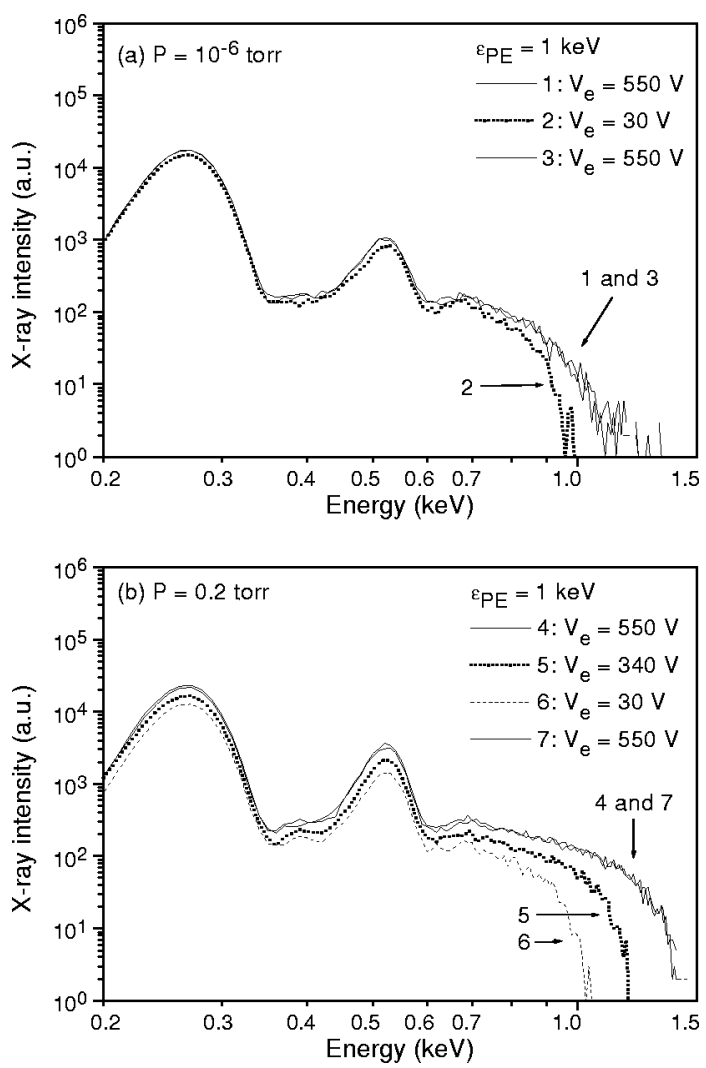

FIG. 4. X-ray spectra acquired consecutively from the same region of mica, in the order shown in the figures, as a function of electrode bias: (a) $P=10^{-6}$ Torr and (b) $P=0.2$ Torr. The data illustrate that positive DHL shifts scale with $V_{e}$ and that irradiation-induced sample modification was not observed when $V_{e}$ was repeatedly cycled between 550 and $30 \mathrm{~V}$ (electron detector $=\mathrm{GSED}, \epsilon_{\mathrm{PE}}=1 \mathrm{keV}, D=10 \mathrm{~mm}, d=4.5 \mathrm{~mm}$, horizontal field width $=130 \mu \mathrm{m}$, and $t_{\text {acq }}=2000$ live s per spectrum).

cept for spectral series obtained under conditions selected so as to maximize these effects for illustrative purposes, as is discussed in Sec. IV C).

The presented results were obtained from muscovite mica specimens, approximately $1 \times 1 \mathrm{~cm}$ wide and $300 \mu \mathrm{m}$ thick. Qualitatively the same behavior was observed in data acquired from sapphire and polytetrafluoroethylene (PTFE).

\section{RESULTS AND DISCUSSION}

\section{A. Electric fields in a low vacuum SEM chamber}

Figure 3 shows $\mathrm{x}$-ray spectra acquired consecutively from the same region of mica using electron beam accelerating voltages of $4,4,10$, and $25 \mathrm{kV}$ and a gas pressure of 0.5 Torr. An axisymmetric ring electrode (the GSED electron collector) biased to $500 \mathrm{~V}$ was located $5.5 \mathrm{~mm}$ above the sample surface during spectrum acquisition. At $\epsilon_{\mathrm{PE}}=4,10$, and $25 \mathrm{keV}$, the PE landing energy, DHL, was approximately 4.4, 10.1, and $25 \mathrm{keV}$, corresponding to positive DHL shifts of 400,100 , and $0 \mathrm{eV}$, respectively. ${ }^{24}$ The $400 \mathrm{eV}$ DHL shift observed at $\epsilon_{\mathrm{PE}}=4 \mathrm{keV}$ did not change with irradiation time, as is seen in the figure (the acquisition time, $t_{\text {acq }}$, was 2000 live $s$ per spectrum).

The dependence of the PE landing energy on the ring electrode bias, $V_{e}$, is shown in Fig. 4 (multiple spectra acquired under identical conditions show the absence of time- 


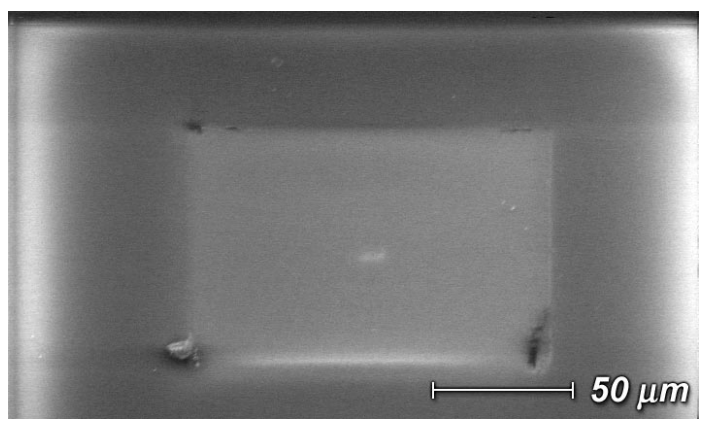

FIG. 5. GSED image of a region (100 $\mu \mathrm{m}$ wide, bright rectangle) of mica preirradiated during the acquisition of an x-ray spectrum that exhibited a positive DHL shift. The image shows SE contrast caused by negative sample charging $\left(\epsilon_{\mathrm{PE}}=4 \mathrm{keV}, P=0.2\right.$ Torr, $D=10 \mathrm{~mm}, d=4.5 \mathrm{~mm}$, and $\left.V_{e}=550 \mathrm{~V}\right)$.

dependent changes caused by irradiation-induced sample modification). Under conditions of both high and low vacuum $\left(10^{-6}\right.$ and 0.2 Torr, respectively), the magnitude of the positive DHL shift increased with $V_{e}$. At a given bias, the DHL shift was much greater under conditions of low vacuum $\left(P \gg 10^{-6}\right.$ Torr), when a relatively large concentration of ionized gas molecules was present in the specimen chamber.

In a low vacuum SEM chamber, the rate at which ions are generated in the gas cascade scales with $V_{e} \cdot{ }^{16,20}$ Consequently, at 0.2 Torr, the steady state concentration of positive ions also scales with $V_{e} \cdot{ }^{12}$ The positive DHL shifts shown in Fig. 4(b) may therefore be contributed to by (i) a "cloud" of positive charge (ions) with a vertical center of gravity located above the sample surface, as shown in Fig. 2, and (ii) positive sample charging caused by recombination of ions with electrons in the vicinity of the sample surface ${ }^{25,26}$ Evidence against the latter is provided by the GSED image shown in Fig. 5. The image shows a region $(100 \mu \mathrm{m}$ wide, bright rectangle) of mica from which an x-ray spectrum was acquired (prior to GSED image acquisition) using an accelerating voltage of $4 \mathrm{kV}$. The spectrum exhibited a positive DHL shift of a few hundred electron volts. However, the bright rectangle corresponds to enhanced SE emission caused by negative sample charging (i.e., a net negative surface potential generated by the bilayer of excess holes and electrons trapped below the sample surface). ${ }^{27-30} \mathrm{~A}$ detailed analysis of methods used to identify contrast caused by negative charging of dielectrics in a low vacuum environment has been presented elsewhere. ${ }^{11}$

The above-mentioned effects of negative sample charging on SE emission were also observed in images obtained during (not only after) the acquisition of spectra that exhibited positive DHL shifts. These data show that an increase in the PE landing energy can be observed under conditions of negative sample charging (here, the polarity of "specimen charging" is defined by the effects of trapped charge on SE emission). That is, under such conditions (i.e., when subsurface trapped charge causes an increase in the SE yield), the positive DHL shift is not caused by positive specimen charging, but by the electric field generated by ionized gas molecules located above the sample surface, as shown in Fig. 2.
However, the (relatively small) DHL shifts observed in spectra acquired in high vacuum, as a function of $V_{e}$ [Fig. 4(a)] indicate that ions cannot be the sole cause of the observed shifts. The shifts may also be contributed to by the effects of the electric field generated by the biased ring electrode on the primary electron beam. The electric field generated by the biased electrode terminates on the specimen stage, not on the surface of an insulating sample (Fig. 2). Hence as a PE travels from the electron gun to the ring electrode, it gains $V_{e}$ electron volts of kinetic energy, but it loses a smaller amount of energy during transit from the electrode to the insulating sample surface. It therefore impacts the surface with an energy greater than $\epsilon_{\mathrm{PE}}$, thus contributing to the net DHL shift (i.e., the electric field generated by the biased ring electrode affects the DHL via the same mechanism as the field generated by the ions).

It should be noted that, unlike the data shown in Fig. 3 and 5, the spectra in Fig. 4 were acquired using an accelerating voltage of $1 \mathrm{kV}$, that is, under conditions where the current of electrons emitted from the sample is greater than the primary beam current (this condition is only satisfied at the start of electron irradiation, before the charge state of the sample converges to a dynamic equilibrium whereby the emissive and primary currents are equal). ${ }^{29}$ Under such conditions, the observed relationship between DHL shifts and $V_{e}$ may also be contributed to by positive sample charging. As was mentioned earlier, conventional (high vacuum) SEM theory states that the magnitude of shifts due to positive sample charging is self-limited to a few electron volts due to pinning of SEs at the specimen surface by the field generated by excess holes. ${ }^{13}$ However, under the specimen-ring electrode geometry employed in this work, the net electric field at the sample surface consists of three components generated by excess charge trapped in the sample, the biased electrode, and ionized gas molecules (if gas is present in the chamber). The latter two components give rise to an SE extraction potential ${ }^{11}$ that opposes the action of the field responsible for SE pinning at the surface. It therefore seems reasonable to argue that the greater the electrode bias, $V_{e}$, the higher the intensity of the field required for SE pinning (caused by positive sample charging) and, consequently, the greater the maximum possible magnitude of positive sample charging. The field generated by positive specimen charging accelerates PEs as they approach the sample surface and contributes to positive DHL shifts.

We will now discuss the decrease in the magnitude of the positive DHL shift observed in spectra acquired as a function of increasing $\epsilon_{\mathrm{PE}}$ as shown in Fig. 3. An increase in $\epsilon_{\mathrm{PE}}$ causes an increase in the maximum $\mathrm{PE}$ penetration range. ${ }^{13}$ At accelerating voltages in excess of approximately $1 \mathrm{kV}$, the greater the penetration range, the lower the SE yield (the mean number of SEs emitted per PE), the larger the maximum depth at which excess electrons are trapped in the sample, and the greater the magnitude of the maximum surface potential caused by negative sample charging. ${ }^{13}$ The electric field generated by excess charge extends beyond the sample surface, into the SEM chamber, and its magnitude increases with increasing $\epsilon_{\mathrm{PE}} \cdot{ }^{13}$ The field decelerates PEs as they approach the sample surface, and thereby reduces the 


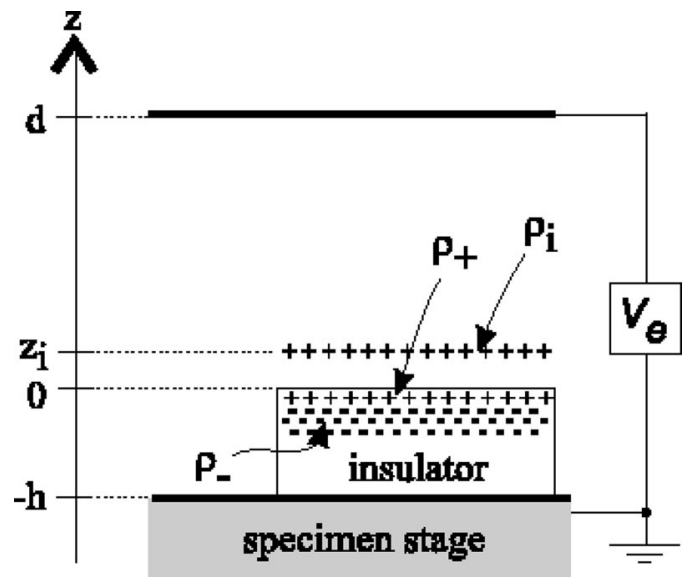

FIG. 6. Schematic illustration of the sources of electric field components in a low vacuum SEM chamber: $V_{e}$ : bias applied to the gaseous electron detector electrode, $\rho_{i}$ : charge density within the plane representing the field generated by ionized gas molecules, $\rho_{+}$: charge density of the positive near-surface layer containing an excess concentration of holes as a result of SE emission, and $\rho_{-}$: charge density of the negative underlayer containing excess electrons injected into the sample.

PE landing energy. This oversimplified line of reasoning can qualitatively account for the observed dependence of the DHL shift on $\epsilon_{\mathrm{PE}}$. A more realistic model needs to account for the interdependence of (i) the intensity and spatial distribution of the net electric field between the sample surface and the ring electrode and (ii) the corresponding generation rate of ions in the gas cascade. The latter affects the steady state ion concentration, ${ }^{12,23}$ the extent of sample charging, ${ }^{1,7}$ and the SE yield. ${ }^{11}$ In contrast to existing models of low vacuum SEM, the charge states of the partially ionized gas and of the specimen should be considered as interdependent components of a single system. The development of such a model is beyond the scope of the present article.

In summary, the PE landing energy in a low vacuum SEM can be expressed as

$$
\mathrm{DHL}=\epsilon_{\mathrm{PE}}+e\left(\Delta V_{5}+\Delta V_{\text {ring }}+\Delta V_{s}\right),
$$

where $e \Delta V_{5}$ is the DHL increase caused by the passage of PEs through the ion cloud [see Fig. 2 and Eq. (1)], $e V_{\text {ring }}$ is the increase caused by the transit of PEs through the ring electrode, and $e V_{s}$ is the change in the landing energy caused by sample charging. The sign of the latter is governed by the polarity of the net electric field generated at the sample surface by subsurface trapped charge. The magnitude of $e V_{\text {ring }}$ is a function of specimen capacitance.

\section{B. Field assisted SE emission from insulators}

The vertical distribution of the different electric field sources discussed above is schematically illustrated in Fig. 6 where $V_{e}$ is the bias applied to the ring electrode, $\rho_{i}$ is the charge density within the plane that represents the vertical distribution of gaseous ions (the height of $\rho_{i}$ above the sample surface, $z_{i}$, is not known), $\rho_{+}$represents the charge density within the positive near-surface layer caused by SE emission (this layer is, in general, tens of nanometers thick), ${ }^{27-30}$ and $\rho_{-}$represents the charge density within the negative underlayer caused by the current injected into the
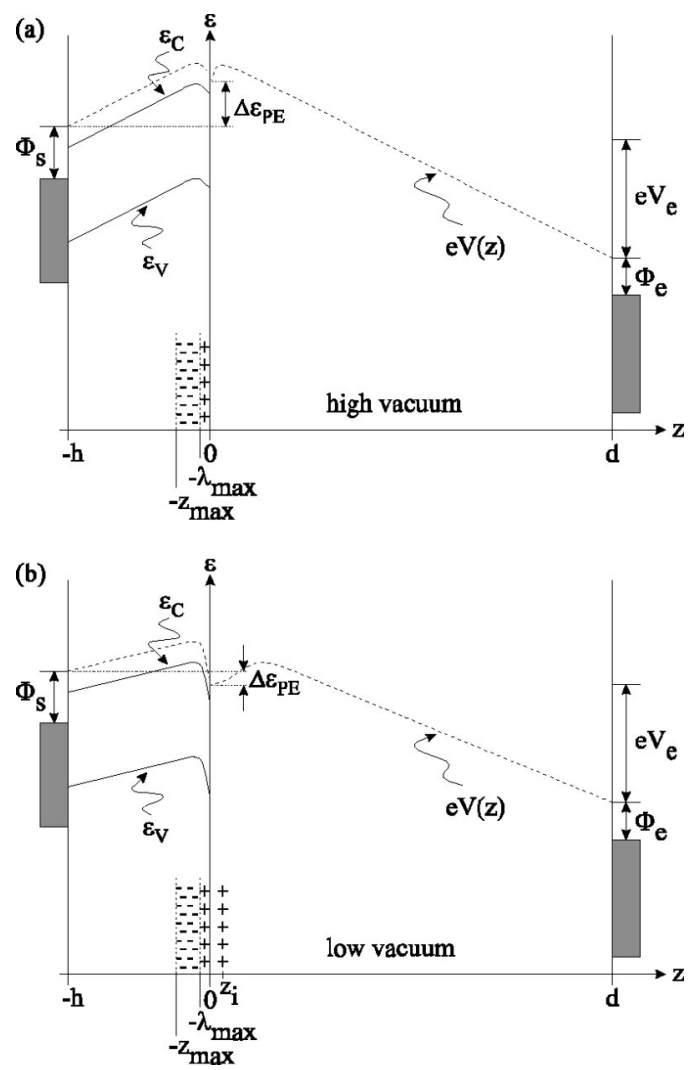

FIG. 7. Simplified electron energy diagram of the stage-sample-biased electrode geometry shown in Fig. 6 (not to scale), under conditions of: (a) high vacuum, negative sample surface potential (adapted from Ref. 30) and (b) low vacuum, positive surface potential $\left(\Phi_{s}\right.$ : specimen stage work function, $\epsilon_{c}$ : specimen conduction band minimum, $\epsilon_{v}$ : specimen valence band maximum, $\Delta \epsilon_{\mathrm{PE}}$ : change in the PE landing energy caused by the sample surface potential, $\Phi_{e}$ : electrode work function, $V_{e}$ : electrode bias, $\lambda_{\max }$ : maximum SE escape depth, $z_{\max }:$ maximum PE penetration range, and $e$ : charge of an electron).

specimen (the underlayer extends down to the maximum PE penetration range). ${ }^{27-30}$ The exact vertical distributions of $\rho_{+}$ and $\rho_{-}$are not known. At beam energies in excess of approximately $1 \mathrm{keV}$, the magnitudes of $\rho_{+}$and $\rho_{-}$generally decrease and increase with increasing PE energy, respectively. ${ }^{27-30}$

Shown in Fig. 7 is a simplified electron energy diagram of the stage-sample-biased electrode geometry shown in Fig. 6 , under conditions of (a) high vacuum, negative surface potential [Fig. 7(a), adapted from Ref. 30] and (b) low vacuum, positive surface potential [Fig. 7(b)]. We emphasize that the exact charge distributions (below and above the sample surface) are not known, and the diagrams shown in Fig. 7 are merely approximations consistent with experimental facts (i.e., the potential at the two electrodes and at the sample surface, the presence of positive and negative layers of charge below the sample surface, ${ }^{30}$ and the presence of gaseous ions above the sample surface).

We shall now consider the effects of these charge distributions on the emission probability of SEs excited by the electron beam. The net field generated by $V_{e}$ and $\rho_{i}$ attracts electrons in the specimen, thereby giving rise to an SE extraction potential at the sample surface. The extraction potential lowers the surface barrier and increases the emission 
probability of low energy SEs (a detailed discussion of the effects of such an extraction potential on emission of SEs from a dielectric can be found in Ref. 11). That is, the greater the positive DHL shift caused by $\Delta V_{5}$ [see Eq. (2)], the greater the SE yield. However, the ion concentration, the source of $\Delta V_{5}$, also affects the SE-ion recombination rate (the rate at which SEs are captured by gaseous ions). ${ }^{23}$ The greater the ion concentration, the greater the SE-ion recombination rate, the smaller the number of SEs amplified in the gas cascade and the greater the suppression of the SE imaging signal induced in the ring electrode. ${ }^{23}$ Hence the net effect of the ions on the SE imaging signal depends on the relative magnitude of these two effects (field-enhanced SE emission and SE-ion recombination), and is a function of SEM operating parameters. ${ }^{11,23}$

The relative magnitudes of the electric field components generated by $\rho_{+}$and $\rho_{-}$govern the net surface potential caused by sample charging. ${ }^{27-29}$ The positive and negative layers serve to decrease and increase the SE emission probability, respectively, ${ }^{11}$ as in the well documented case of high vacuum SEM. ${ }^{27-29}$

We should reemphasize that the magnitudes of field components generated by $\rho_{i}, \rho_{+}$, and $\rho_{-}$are interdependent and, when the electron beam is initially switched on, the final charge state that the partially ionized gas-dielectric system converges to depends on the cascade (i.e., ion generation) characteristics of the gas, charge states, and species of gaseous ions (which affect the ion neutralization rates), dielectric properties of the sample, microscope operating parameters (e.g., $\epsilon_{\mathrm{PE}}, V_{e}, P, d$, beam current, scan speed, and magnification) and, as is discussed below, the geometry and electrical properties of objects in the specimen chamber.

\section{Radiation induced sample modification}

It is well known that electron irradiation of a dielectric in a high vacuum SEM can lead to sample modification due to filling of charge traps with excess electrons or holes, irradiation-induced defect formation, electromigration of defects and impurities under the influence of the electric field produced by trapped charge, and adsorption/desorption processes at the sample surface. ${ }^{30-34}$ Bombardment of dielectrics by soft-landing ions can also modify the surface via ion adsorption/desorption processes. ${ }^{26}$ All of these phenomena can, in principle, cause time-dependent changes in the amount of charge trapped in an insulator in a low vacuum SEM which can, in turn, affect the PE landing energy. Such changes are illustrated by the $\mathrm{x}$-ray spectra, acquired (from the same area) as a function of irradiation time, shown in Fig. 8. The spectra show a time dependent decrease in the magnitude of the positive DHL shift from approximately 400 to $0 \mathrm{eV}$. Such behavior was most pronounced under conditions of high electron beam current. The specific causes of these results are not relevant to the present discussion. The data have been included to demonstrate the existence of such dynamic processes in low vacuum SEM and to point out that all other DHL shifts discussed were not caused by irradiation-induced sample modification, as is emphasized throughout the article.

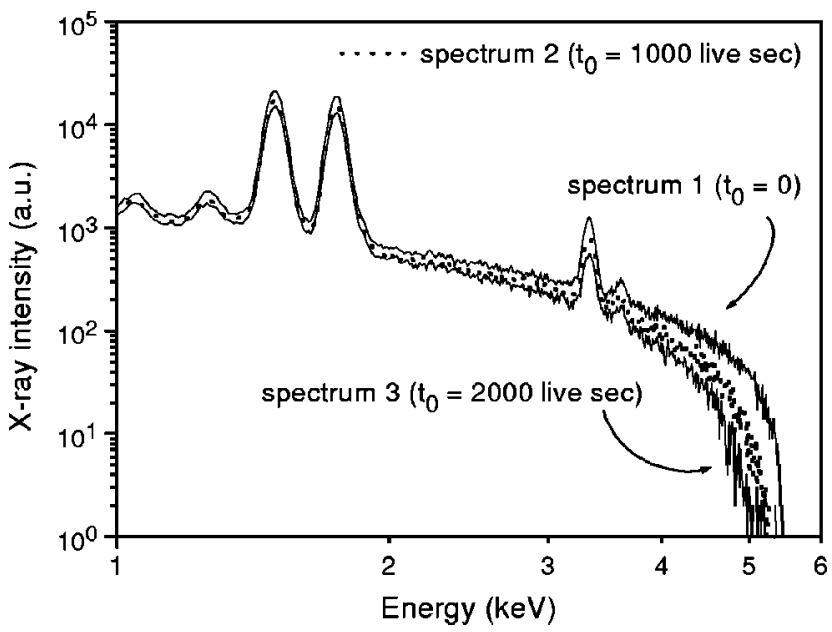

FIG. 8. X-ray spectra acquired consecutively from the same region of mica. The spectra show changes in the PE landing energy, DHL, as a function of irradiation time (electron detector $=\mathrm{GSED}, \epsilon_{\mathrm{PE}}=5 \mathrm{keV}, P=0.2$ Torr, $D=10 \mathrm{~mm}, d=4.5 \mathrm{~mm}$, horizontal field width $=130 \mu \mathrm{m}$, and $t_{\text {acq }}=1000$ live $s$ per spectrum).

\section{Consequences for low voltage x-ray microanalysis}

The intensity of a characteristic $\mathrm{x}$-ray line is highly dependent on the PE landing energy. ${ }^{13}$ The changes in the landing energy indicated by the positive DHL shifts observed in $\mathrm{x}$-ray spectra of dielectrics in a low vacuum environment can therefore introduce errors into $\mathrm{x}$-ray quantification procedures. Such artifacts can be alleviated by adjusting operating parameters such as $V_{e}$ and $P$ so as to minimize the positive DHL shifts. However, on the basis of the proposed electric field model, a more elegant solution is the employment of sample-electrode geometries in which the steady state ion concentration is minimized, and DHL shifts caused by the transit of PEs past the biased electrode are minimized. Two such geometries are schematically illustrated in Fig. 1: (i) a thin grounded conductor, such as a series of parallel wires, is placed between the ring electrode and the sample surface, ${ }^{18}$ or (ii) the axisymmetric ring electrode is replaced by a plate electrode, located off-axis, near the pole piece. Twodimensional approximations to the distributions of electric equipotentials generated by the positively biased electrodes $\left(V_{e}=500 \mathrm{~V}\right)$ arranged in these geometries are shown in Figs. 9(a) and 9(b), respectively. The calculations were performed using the finite element software QuickField. ${ }^{35} \mathrm{X}$-ray spectra obtained using these geometries, acquired as a function of $V_{e}$, are shown in Fig. 10. For comparison, the figure also contains spectra obtained using the ring electrode geometry employed during the acquisition of all other spectra presented in this article (i.e., in the absence of the grounded wires), and a spectrum obtained from one of the grounded wires. The latter shows the expected absence of DHL shifts in x-ray spectra of grounded conductors (under all conditions).

The spectra in Fig. 10(a) show that the presence of grounded wires below the ring electrode causes a significant decrease in the magnitude of the positive DHL shift in x-ray spectra of insulators. The wires provide termination points for some fraction of the electric field produced by the ring 

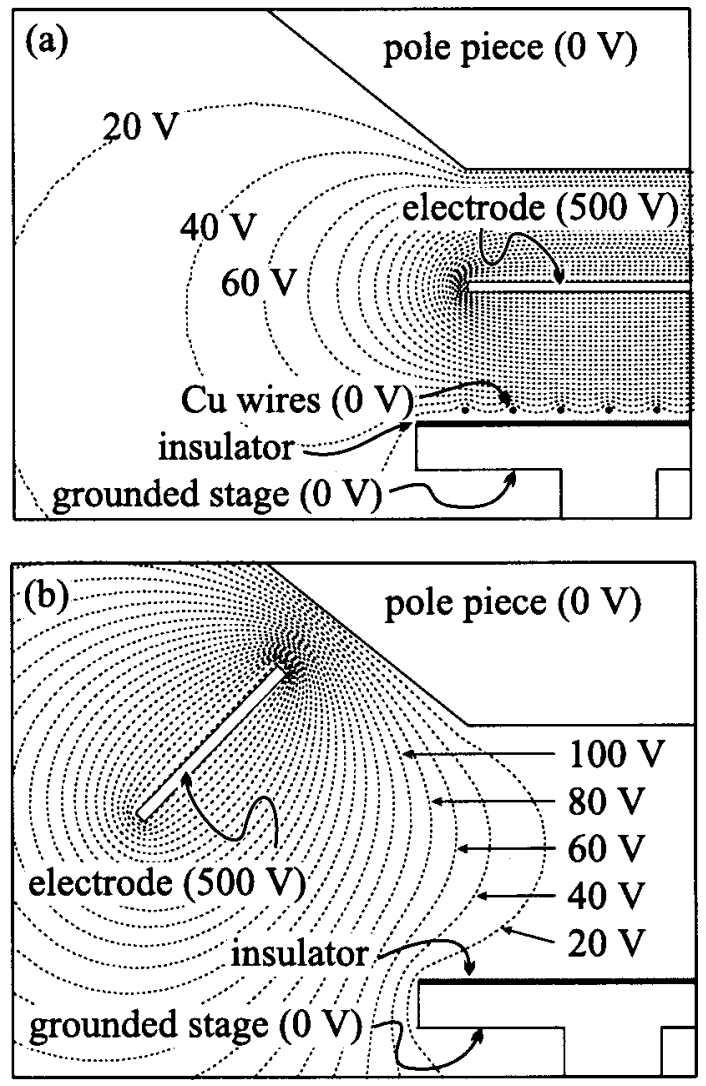

FIG. 9. Distributions of electric equipotentials (broken lines, $\Delta V=20 \mathrm{~V}$ ) in a low vacuum SEM chamber calculated for simplified geometries corresponding to: (a) GSED electron detector and an array of grounded wires located above the sample and (b) LFGSED electron detector (see Fig. 1).

electrode, ${ }^{18}$ as is illustrated by the equipotential plot shown in Fig. 9(a). The presence of the wires therefore causes a reduction in $e \Delta V_{\text {ring }}$, the increase in DHL caused by the passage of PEs through the ring electrode [see Eq. (2)].

The wires also affect the electric field generated by gaseous ions. The steady state ion concentration is governed by the ion generation and neutralization rates. ${ }^{23}$ The latter is much greater in the case of grounded conductors than in the case of insulators. ${ }^{12,22,23}$ Consequently, the wires also serve to reduce the steady state concentration of ions above the surface of a dielectric ${ }^{18}$ and, consequently, $e \Delta V_{5}$, the increase in DHL caused by the passage of PEs through the ion cloud [see Fig. 2 and Eq. (2)].

However, the spectra shown in Fig. 10(a) illustrate that, even when the wires are present in the chamber, DHL is greater than $\epsilon_{\mathrm{PE}}$, and it scales with $V_{e}$. These small DHL shifts are caused by the small fraction of the detector field that extends beyond the wires [see Fig. 9(a)] and the field generated by residual ions, the presence of wires above the sample reduces, but does not eliminate the concentration of ions above the specimen surface. This shift can therefore be minimized by optimizing the distance between the wires and the sample surface, and by optimizing the interwire separation.

An alternate solution is to employ an off-axis electrode located near the pole piece, as shown in Fig. 9(b). In this case, as is seen in the figure, the electric field between the
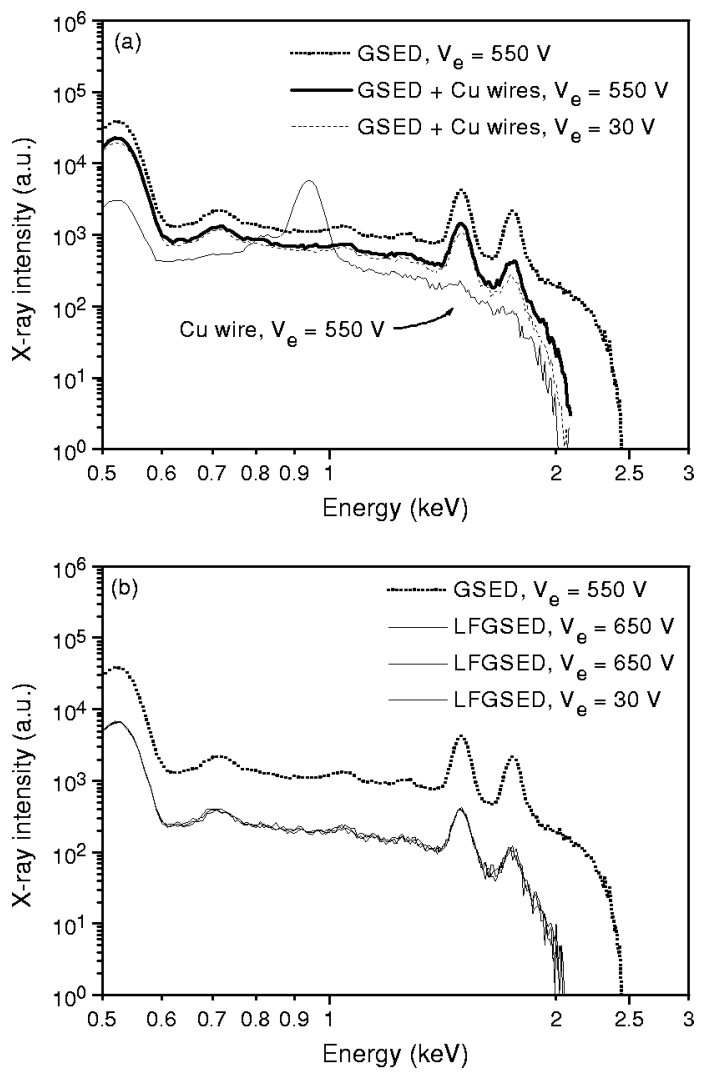

FIG. 10. X-ray spectra of mica acquired using the sample-detector geometries defined in Figs. 1 and 9. The spectra show the dependence of the PE landing energy, DHL, on the distribution of electric field lines in the specimen chamber. Data obtained under identical conditions show the absence of irradiation induced changes in x-ray spectra $\left(\epsilon_{\mathrm{PE}}=2 \mathrm{keV}, P=0.5\right.$ Torr, $D=10 \mathrm{~mm}, \quad d=4.5 \mathrm{~mm}, \quad w=0.5 \mathrm{~mm}, \quad$ interwire separation $=2 \mathrm{~mm}$, horizontal field width $=130 \mu \mathrm{m}$ (except for the spectrum obtained from a grounded $\mathrm{Cu}$ wire which was acquired in spot mode), and $t_{\mathrm{acq}}=2000$ live $\mathrm{s}$ per spectrum).

pole piece and the sample (generated by $V_{e}$ ) is very weak. Consequently, the increase in PE landing energy caused by the passage of PEs past the electrode is small. The equipotential plot also shows that some fraction of the field terminates on the pole piece. Hence ions generated in the gas cascade between the sample and the electrode can drift to, and be neutralized during contact with, the pole piece. The concentration of ions above the sample surface and the magnitude of $\Delta V_{5}$ are therefore self-limited since, if the ion concentration increases, the corresponding electric field efficiently repels subsequently generated ions away from the specimen, towards the pole piece (since the detector field at the surface is very weak) where the ions are rapidly neutralized.

If the sample exhibits negative charging, the electric field generated by excess electrons attracts ions to the sample surface, and the field generated by these ions cancels the field (in the gas, above the sample) generated by the electrons trapped in the specimen. Hence DHL shifts caused by negative sample charging are minimized. Any subsequent buildup of excess ions above the (negatively charged) sample is again self-limited (under the GSED $+\mathrm{Cu}$ wire geometry, this argument also applies to the concentration of ions between the sample surface and the wires). 
If the sample exhibits positive charging, the electric field (above the sample) generated by excess holes (located in the sample) repels ions generated in the gas cascade and therefore limits the magnitude of $\Delta V_{5}$. The low intensity of the detector field at the sample surface and the self-limited ion concentration also serve to limit the maximum allowed surface potential caused by positive sample charging. DHL shifts caused by the field generated by excess holes are therefore also minimized.

The effectiveness of the off-axis electrode geometry in reducing DHL shifts is clearly illustrated by the spectra shown in Fig. 10(b).

\section{CONCLUSION}

$\mathrm{X}$-ray spectroscopy and SE imaging were used to illustrate the effects of electric fields in a variable pressure SEM chamber generated by ionized gas molecules and by excess charge trapped in dielectric specimens. The results provide experimental evidence for the electric field distribution recently proposed in a model of charge-induced SE emission from dielectrics in a low vacuum environment. ${ }^{11}$ It was demonstrated how field-induced x-ray microanalysis artifacts can be minimized by use of appropriate sample-electrode-pole piece geometries.

\section{ACKNOWLEDGMENTS}

The authors gratefully acknowledge useful discussions with Debbie Stokes, Archie Howie, and Frank Baker. This work was funded by the EPSRC (Grant No. GR/M90139) and FEI corporation.

${ }^{1}$ G. D. Danilatos, Adv. Electron. Electron Phys. 71, 109 (1988).

${ }^{2}$ G. D. Danilatos, J. Microsc. 162, 391 (1991).

${ }^{3}$ V. N. E. Robinson, J. Phys. E 8, 638 (1975).

${ }^{4}$ V. N. E. Robinson, Scanning 1, 149 (1978).

${ }^{5}$ P. Meredith and A. M. Donald, J. Microsc. 181, 23 (1996).
${ }^{6}$ D. J. Stokes, B. L. Thiel, and A. M. Donald, Langmuir 14, 4402 (1998).

${ }^{7}$ M. Toth, S. O. Kucheyev, J. S. Williams, C. Jagadish, M. R. Phillips, and G. Li, Appl. Phys. Lett. 77, 1342 (2000).

${ }^{8}$ D. J. Stokes, B. L. Thiel, and A. M. Donald, Scanning 22, 357 (2000).

${ }^{9}$ T. C. Baroni, B. J. Griffin, J. R. Browne, and F. J. Lincoln, Microsc. Microanal. 6, 49 (2000).

${ }^{10}$ B. J. Griffin, Scanning 22, 234 (2000).

${ }^{11}$ M. Toth, M. R. Phillips, B. L. Thiel, and A. M. Donald, preceeding paper, J. Appl. Phys. 91, 4479 (2002).

${ }^{12}$ M. Toth and M. R. Phillips, Scanning 22, 319 (2000).

${ }^{13}$ L. Reimer, Scanning Electron Microscopy (Springer, Berlin, 1985).

${ }^{14}$ D. A. Moncrieff, P. R. Barker, and V. N. E. Robinson, J. Phys. D 12, 481 (1979).

${ }^{15}$ E. Doehne, Scanning 19, 75 (1997).

${ }^{16}$ G. D. Danilatos, Adv. Electron. Electron Phys. 78, 1 (1990).

${ }^{17}$ XL30 ESEM FEG SEM Operating Instructions, FEI Company, Boston, 2000.

${ }^{18}$ J. P. Craven, F. S. Baker, B. L. Thiel, and A. M. Donald, J. Microsc. 205, 96 (2002).

${ }^{19}$ D. A. Moncrieff, V. N. E. Robinson, and L. B. Harris, J. Phys. D 11, 2315 (1978).

${ }^{20}$ B. L. Thiel, I. C. Bache, A. L. Fletcher, P. Meredith, and A. M. Donald, J. Microsc. 187, 143 (1997)

${ }^{21}$ M. R. Phillips, M. Toth, and B. J. Griffin, Microsc. Microanal. 6, 786 (2000).

${ }^{22}$ M. Toth and M. R. Phillips, Scanning 22, 370 (2000).

${ }^{23}$ M. Toth, B. L. Thiel, and A. M. Donald, J. Microsc. 205, 86 (2002).

${ }^{24}$ The reported DHL shifts are accurate to approximately $\pm 50 \mathrm{eV}$. Trends such as the discussed $\epsilon_{\mathrm{PE}}, V_{e}$, and $P$ dependencies of the DHL were observed in spectra of all materials investigated in this study (mica, sapphire, and PTFE).

${ }^{25}$ H. D. Hagstrum, Phys. Rev. 122, 83 (1961).

${ }^{26}$ D. W. Vance, J. Appl. Phys. 42, 5430 (1971).

${ }^{27}$ J. Cazaux, J. Appl. Phys. 59, 1418 (1986).

${ }^{28}$ A. Melchinger and S. Hofmann, J. Appl. Phys. 78, 6224 (1995).

${ }^{29}$ J. Cazaux, J. Appl. Phys. 85, 1137 (1999).

${ }^{30}$ J. Cazaux, J. Electron Spectrosc. Relat. Phenom. 105, 155 (1999).

${ }^{31}$ M. A. Stevens Kalceff and M. R. Phillips, Phys. Rev. B 52, 3122 (1995).

${ }^{32}$ O. Jbara, J. Cazaux, and P. Trebbia, J. Appl. Phys. 78, 868 (1995).

${ }^{33}$ O. Jbara, J. Cazaux, G. Remond, and C. Gilles, J. Appl. Phys. 79, 2309 (1996).

${ }^{34}$ M. Toth, K. Fleischer, and M. R. Phillips, Phys. Rev. B 59, 1575 (1999).

${ }^{35}$ QuickField Finite Element Analysis System, Tera Analysis, Svendborg, 2001. 
Journal of Applied Physics is copyrighted by the American Institute of Physics (AIP). Redistribution of journal material is subject to the AIP online journal license and/or AIP copyright. For more information, see http://ojps.aip.org/japo/japcr/jsp

Copyright of Journal of Applied Physics is the property of American Institute of Physics and its content may not be copied or emailed to multiple sites or posted to a listserv without the copyright holder's express written permission. However, users may print, download, or email articles for individual use. 


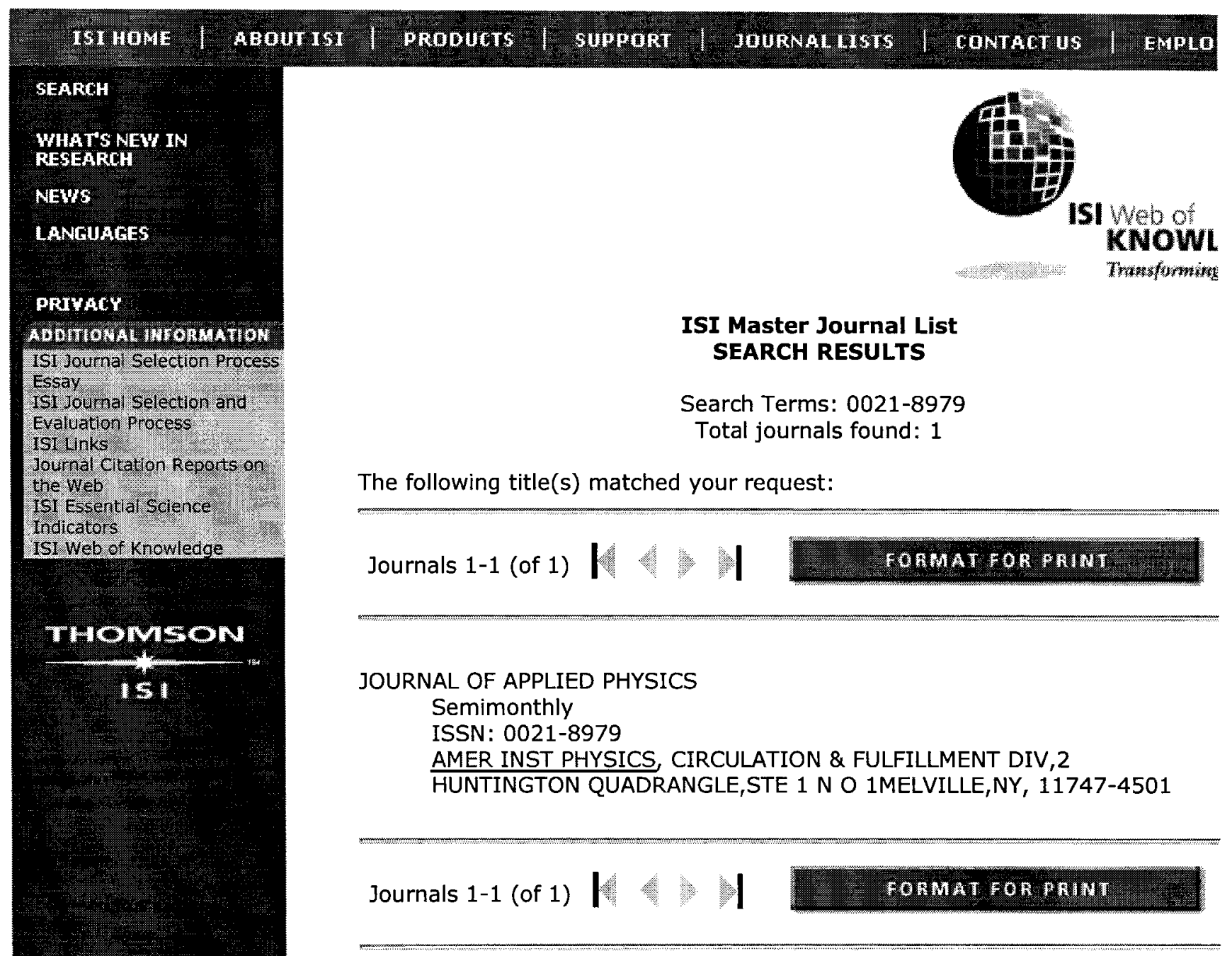

